\title{
ORGANIZING NETWORK INTERACTION IN MODULAR ROBOT With Multilevel ConTrol System
}

\author{
Victor Andreev \& Pavel Pletenev
}
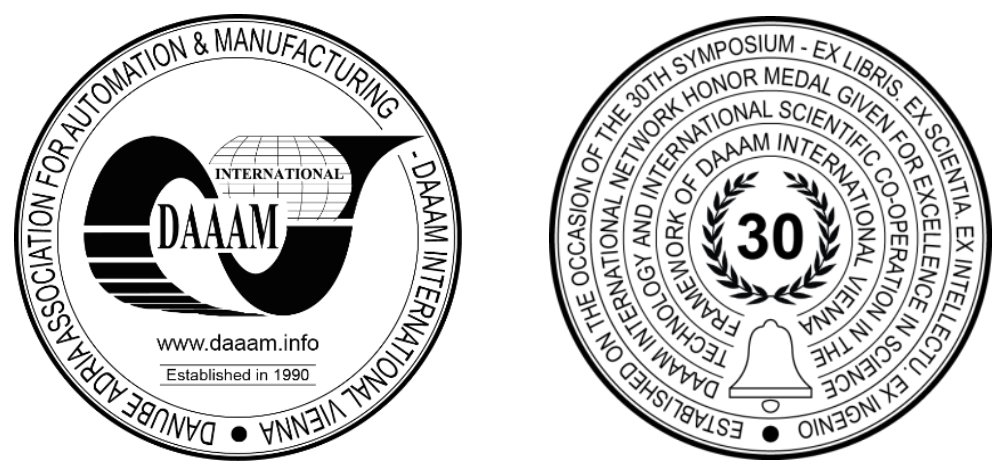

This Publication has to be referred as: Andreev, V[ictor] \& Pletenev, P[avel] (2019). Organizing Network Interaction in Modular Robot with Multilevel Control System, Proceedings of the 30th DAAAM International Symposium, pp.04840492, B. Katalinic (Ed.), Published by DAAAM International, ISBN 978-3-902734-22-8, ISSN 1726-9679, Vienna, Austria

DOI: $10.2507 / 30$ th.daaam.proceedings.065

\begin{abstract}
The features of network communication in mobile modular robots with multilevel control system (CS) are considered both inside single layer and between multiple layers. Modular architecture enables implementation of rapid reconfiguration of robotic systems. Most of modern systems are built as one piece - all data streams come to a single powerful onboard computer, which implements the major part of control system. Hierarchical division on modules and submodules with their own CS enables lowering computational load on central parts of control systems via distributing calculations between them, abstracting and fusing data. Implementing such multilevel modular control system places restrictions on the possible means of communication between modules and submodules. In this paper, communicational bonds in a modular robot are being considered and parameters of emerging information flows are being analyzed. Based on this, the requirements for the types of networks and their topologies are presented.
\end{abstract}

Keywords: modular robot; mobile robot; network technologies; informational interaction; reconfiguration.

\section{Introduction}

The increased use of mobile robots (MR), which is currently observed, leads to an expansion in both algorithmic and memory complexity of the robotic systems (RTS) control systems (CS). This in turn requires higher performance computers, implementing RTS' CS, which is known to be not always possible. Another way is to create an RTS with a modular architecture [1], in which each module is equipped with its own CS, built on the basis of a low-performance systems - embedded computing devices (microcontrollers and microcomputers). Then RTS-wide control and decisionmaking is performed on a distributed computing cluster, lowering the necessary computing power overall. Modular implementation of RTS's CS requires such an intermodule communication organisation that synchronizes operation of all modules. This can be a real problem, which isn't solved to present day in robotics, especially in the context of use of small embedded computing devices.

The second problem is the need for reconfigurability of an RTS. This is due to the obvious need to use MRs where human stay is fraught with danger to their health or life, for example, for work in extreme conditions of the Arctic and Antarctic, space and in solving the problems of the Ministry of emergency situations. In these conditions, robots with the necessary functionality should be assembled in the place of the carried-out works and during them. 
Therefore, the reconfiguration and/or scaling process should be as simple as possible. It should be performed in the "plug and play" fashion. Therefore there is a need for the rapid and automatic reconfiguration of the CS. As shown in [2], and this problem is solved by creating robots with modular architecture of CS. The CS should consist of modules, communicating using predefined rules and exposing an application programming interface (API). It is necessary to develop a well-defined specification on both rules and API to ensure interoperability of modules of different manufacturers.

It is proposed to consider the structure of the MR control system (in the minimum version) as a synergetic union of full-featured modules (Fig.1): transport module - TM, power module - PM, sensor system consisting of short-range module - SRSM and long-range module - LRSM, active interaction modules with the external environment - EIM (manipulators, grippers, etc.) and intelligent control module - ICM. In this structure, each module is responsible for only one function of the robotic system. The principle of full functionality of modules is formulated in [3] as follows: each robot module should be able to perform its goal function in any suitable way, using only its own means to execute commands from an external control system.

Research carried out within the framework of RFBR grant 16-07-00811a "Development of functional-modular principle of construction of hardware and software of intelligent mobile robotic systems" [3] showed that the two-level hierarchy of the modular architecture (see Fig. 1) is unable to provide real-time control process on embedded computing devices, because functional modules CS algorithms are quite complex. The amount of information processed in modules is still large, and the computing power of embedded systems is not always sufficient to provide real-time performance. Especially expensive is the process of processing and integration of sensory information and decision-making at the level of intelligent system-wide control module. As the complexity of the tasks to be solved by MR both in stand-alone and in supervisory mode grow, so the algorithms of the modules' CS will become more complex, and the use of microcomputers with greater performance will still lead to limited functionality of the module.

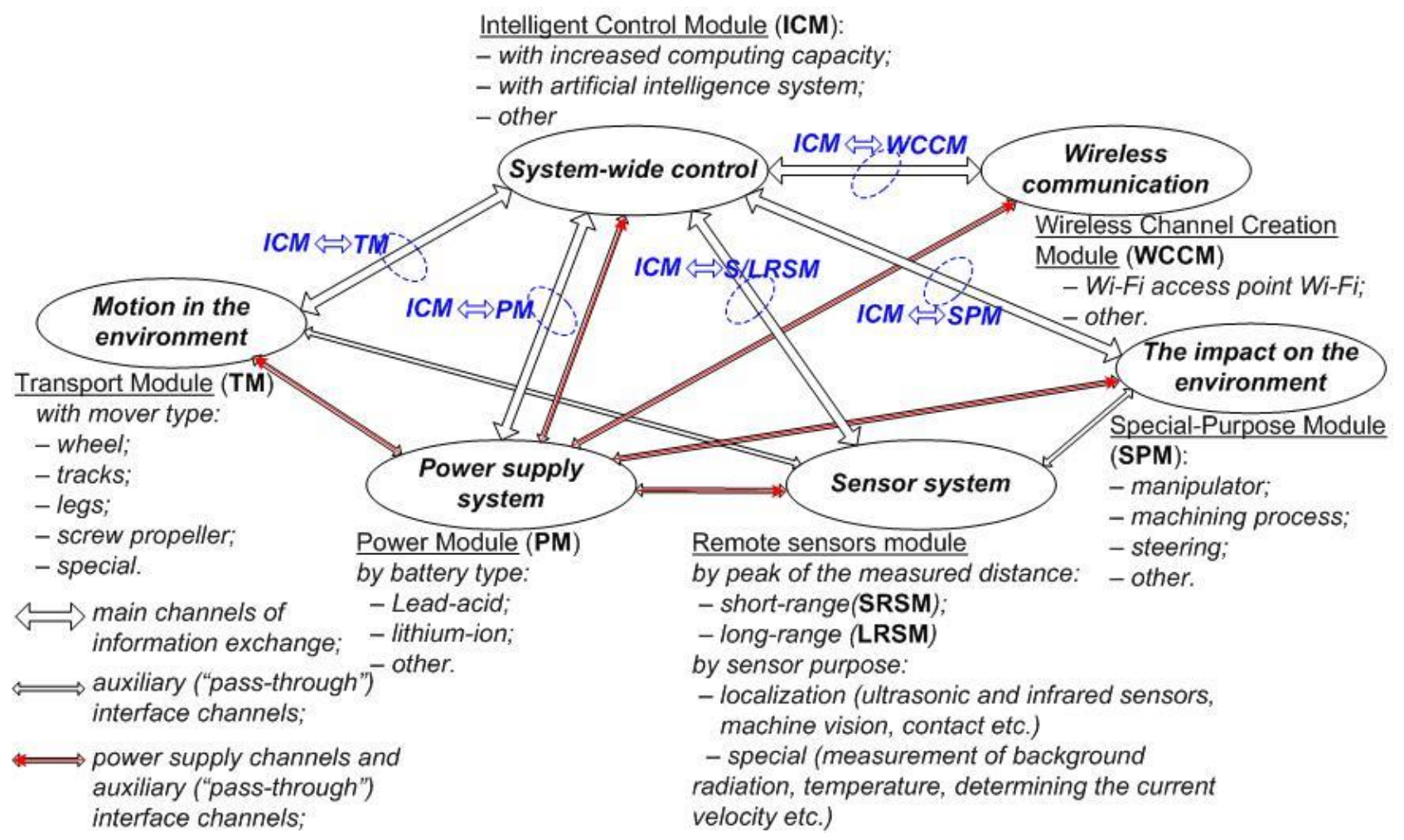

Fig. 1. Functional-modular architecture of information-measuring and control system of the mobile robot.

A number of experiments conducted with the transport module showed the possibility of further development of the MR modular architecture in division the full-featured modules into submodules and creating a multi-level hierarchical (pyramidal) network topology of CS, which ensures the use of embedded computing systems at each level of the hierarchy [5]. This topology significantly simplifies technologies of reconfiguration and scaling of robotic systems, which in turn enables creation of universal modules, lowering robotic systems variety and cost. The proposed solution requires deep research in intermodular network communication, including choice of communication channels, formalization of intermodular communication language etc. Also, one of the main limitations of solutions to the highlighted problem is the necessity to use as full-functional modules and submodules low-power embedded devices, which aren't able to run very complex software due to their limited capabilities. 
The goal of this paper is to present the principles of choice for communication network in a modular robot with hierarchical multilevel CS. Therefore it was necessary to analyse the structure of levels of different possible modules and to synthesize requirements on the communicational network. Then it was needed to analyse modules' and submodules' traffic requirements and existing networks capacities as an example of network choice.

\section{Multi-level subordination modules and submodules}

Within the framework of the given concept, a multi-level subordination of modules and submodules is introduced so that the resulting system is (a) implemented on simple embedded devices and (b) the computational load of overall robot control is distributed among cluster of a variety of different computers. Let us consider this approach on the examples of the main functional modules of MR (see fig. 1): functional blocks of the transport module [6], of the short-range and longrange sensor module [7], and of the power module. The proposed schemes of the modules are shown in fig. 2,3 and 4. At least 3 levels of interaction and subordination can be distinguished in these modules:

- 0th (zeroth) level of interaction (and corresponding "networks" of zeroth level, see fig. 2-4):

$\circ$ primary analysis of sensory data, filtering and conversion to structural representation of information;

○ creating closed-loop drives and other control systems.

- 1st level of interaction (and corresponding First Level Network, see fig. 2,3) - level of full-functional submodules. The following is implemented here:

○ secondary data analysis, aggregation of readings from several submodules;

○ development and distribution of tactical tasks for this module as a whole.

- 2nd level of interaction (and corresponding Second Level Network, see. fig. 2-4) - level of full-functional modules:

$\circ$ tertiary data analysis, aggregation of data from same and different types of modules;

$\circ$ development and distribution of strategic tasks for the robot as a whole.

It is assumed that the interaction of modules with an external supervisor will also occur at the 2 level of interaction.

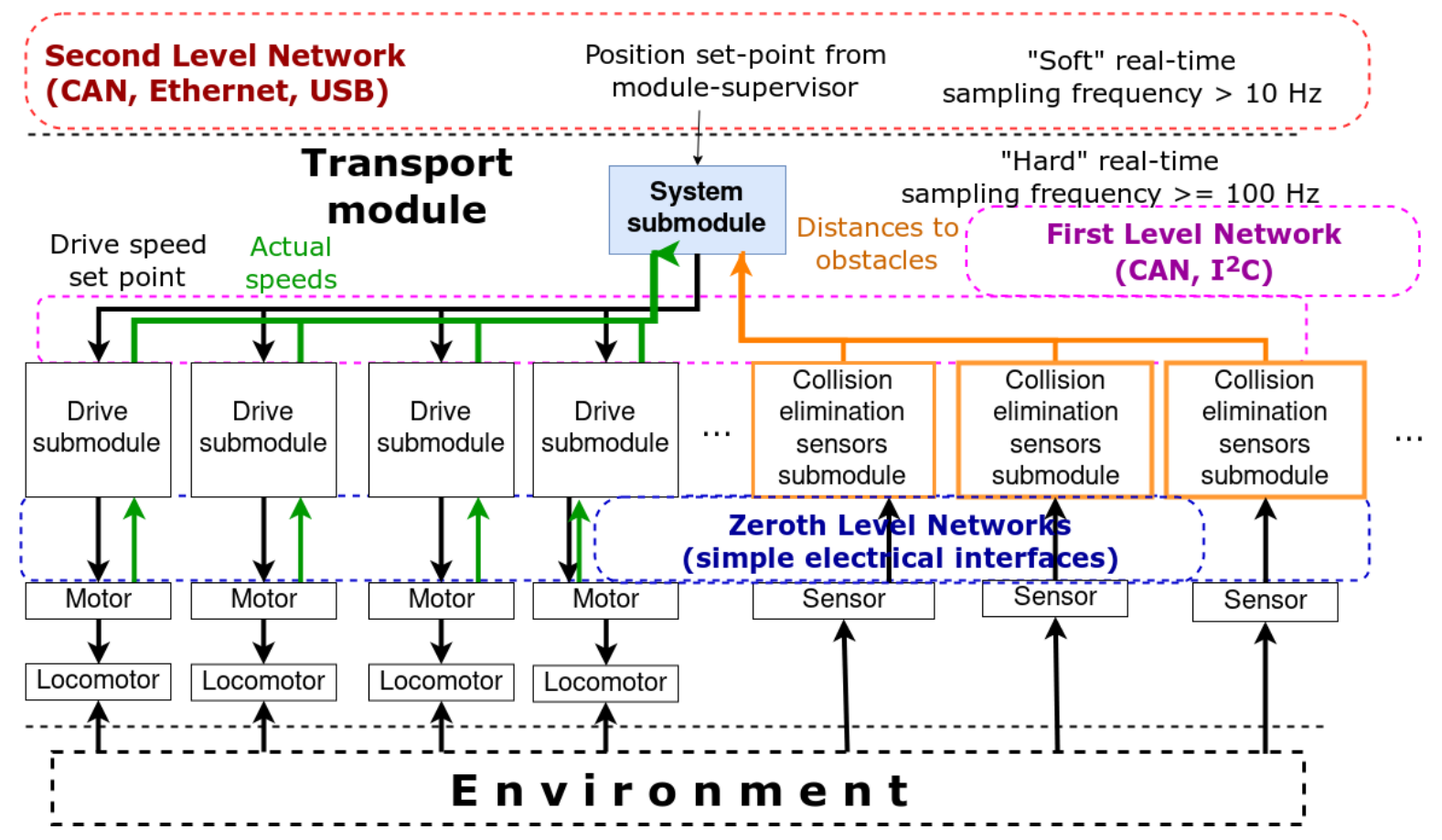

Fig. 2. Transport module's structure [6].

Another, specialized method of interaction is also possible - through the power line. Since it is assumed (see [5]) that all modules will be powered from one shared power line, it is possible to create a communication system between all modules and submodules included in the robot. However, such a communication channel will have low bandwidth.

As one can see in figures 2, 3 and 4, each of the modules and submodules is, in fact, a specialized border router, which converts network traffic of a higher level to a lower level, except when all modules and submodules are connected to one common bus. Also, figure 4 shows the case when the network of the 2 nd level interacts with the networks of the 0 -th level, i.e. the 1st level "collapses" inside one submodule, and the submodule of the generalized control of the power supply performs the tasks of two levels at once, which will require separation in time. 


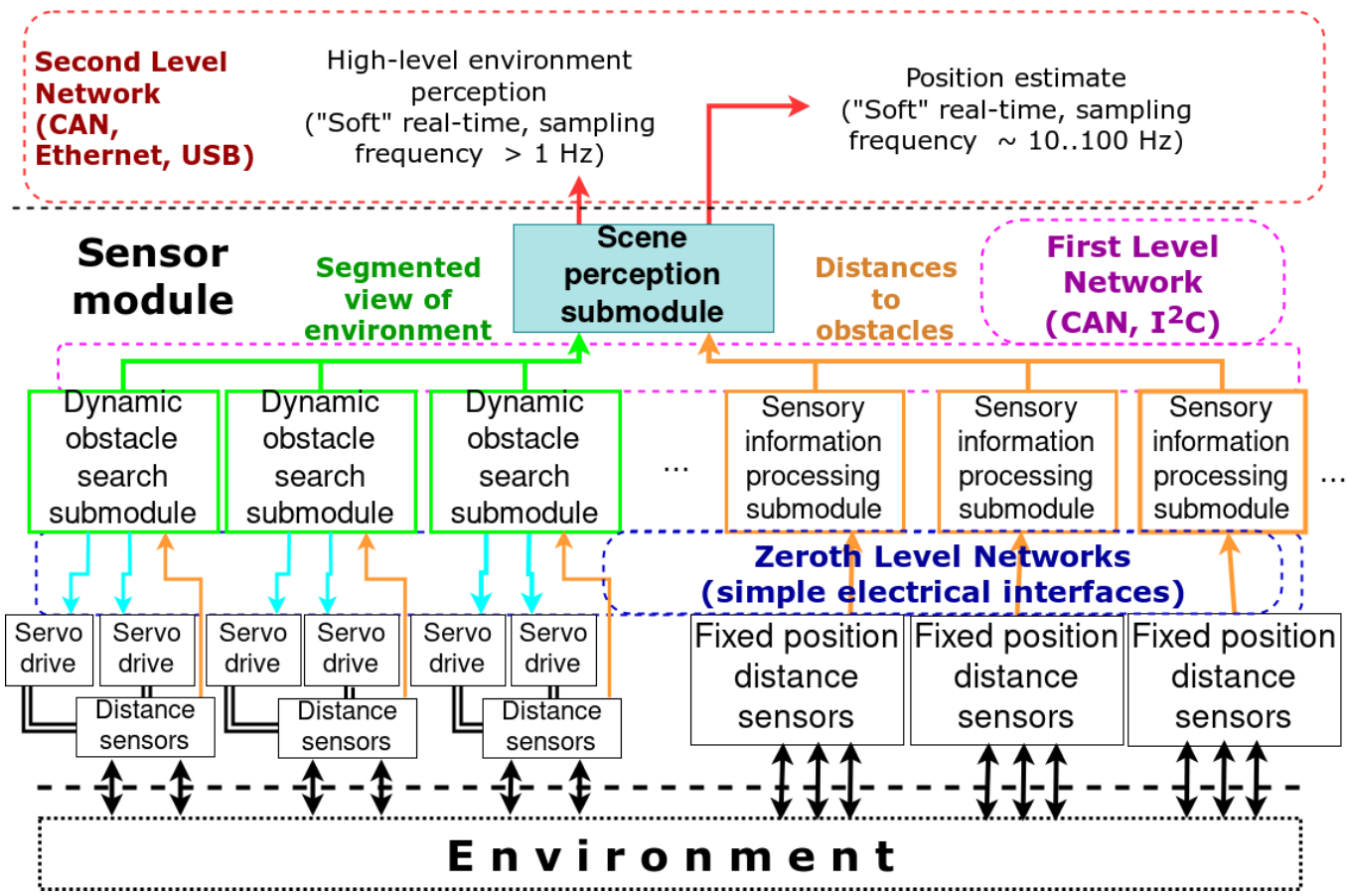

Fig. 3. Sensor module's structure.

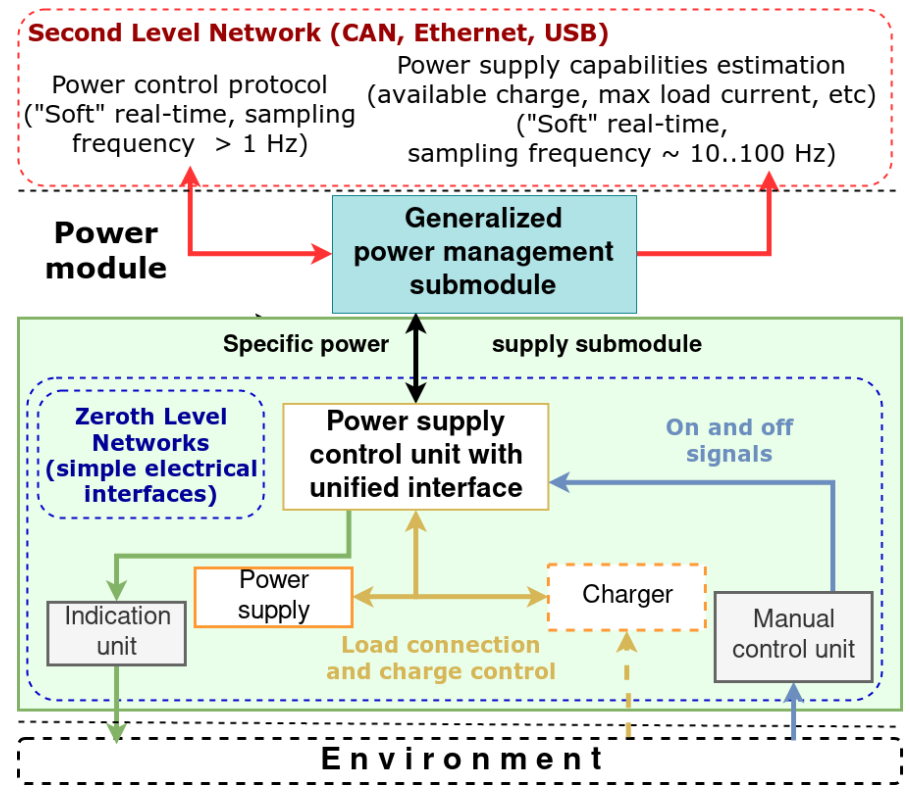

Fig. 4. Power module's structure.

This concept is complicated by the multiplicity of possible technical solutions. In principle, there are no universal solutions for zeroth level networks - each actuator or sensor has its own unique ways of connection and control. Many sensors and actuators can be grouped together, and the ways they interact at the 0 -th level of the network are the details of the implementation of each specific submodule and should not be significant in the organization of intermodule interaction.

First and Second Level Networks are also a part of the implementation of each module, but they generally need to interact with each other, as well as with all possible modules according to general principles, and obey the same set of commands. This is only possible if these networks are standardized.

\section{The use of frameworks and standard libraries}

Existing libraries and frameworks offered by different researchers in [8], [9], [10] and [11] do not allow dynamic reconfiguration. Of particular interest is the approach implemented in the framework of EmsBoT [12], which proposes 
the creation of an abstraction layer over the real-time OS. This layer would allow the same interaction between different networks, providing the same software interface for communication via CAN, Ethernet, USB and other networks, using the same software code and a set of tools for creating agents. Unfortunately, the implementation of this approach presented in the article does not imply dynamic reconfiguration.

From this point of view, we consider the previous work - specification 1/PIMI [13] (Protocol of intermodule interaction) and the method of information interaction for distributed control systems in robots with modular architecture [14]. In these works, the structure of the modular robot IMCS is used, shown in fig. 1. The following was assumed:

- All modules of one type within one robot are available in a single instance and fully perform their own functional purpose.

- All properties of the module and its functional characteristics are given in its meta-information.

The current approach - a modular robot with a hierarchical IMCS topology - is that any type of module can be represented in multiple instances, each of which can consist of several functional submodules. In this case, each of the submodules can be further divided into smaller, possibly incomplete functionally blocks - specific sensors and actuators (drives).

Due to the fact that changing the structure of a modular robot changes different characteristics (mass, moment of inertia, etc.), it is necessary to rebuild and adjust the control system of modules and submodules of robots. Therefore, for the correct operation of the mechanisms for automatic reconfiguration of modules and submodules at all levels, it is necessary to automatically update the knowledge of modules about the relative location of modules and submodules, their size, weight, etc. Therefore, First and Second Level Networks need to provide information about the topology of the modules - information on where exactly a module is attached to or detached from.

The previous specification [13] referred only to Ethernet. This network has shown its not the highest efficiency in terms of module size and complexity of implementation of all levels of abstraction for protocols using TCP/IP stack. This means that further development of the specification needs to be carried out taking into account old and new requirements and limitations. Since the existing communication networks, which are used in various modular robots, differ in physical methods of data transmission and protocols, it is necessary to use the formalism of the OSI model [15] to compare the protocol stacks of communication networks.

1. A communication network must provide data transmission at a speed sufficient for the modules and, where necessary, ensure deterministic delivery of messages.

2. A communication network must not have an explicit master node for the data transmission, i.e., a communication network must ensure the creation of horizontal links.

3. A communication network must be able to provide the node with information about the topology of the connection of other nodes in the network.

4. If one selects multiple networks for one layer, there must be one defined way to convert message of one network type to message of another network type.

5. Standard connectors for network communications, if they are specified by the physical layer of standard of a network, must be as small as possible.

6. As many levels of communication network as possible must be implemented at the hardware level in embedded systems.

7. For most embedded devices, there must be a library (or multiple libraries) to work with a communication network.

8. The physical and/or link layer of the communication network must be noise-proof and/or have error detection and correction mechanisms for transmitting information at distances specific to the modules.

It should be noted that requirements 2 and 3, in fact, contradict each other. Therefore, any choice of network will be a compromise between the ability to create horizontal links and obtain information about the topology and connection points of the nodes. This list of requirements may not be complete, and some items may not be required. The solution of this problem requires further both theoretical and practical research.

\section{Existing communication networks}

At the moment, many robots with modular architecture have been created: homogeneous [16], [17], [18], [19] and heterogeneous [20], [21], [22] and [23]. They use many different types of networks. Each type of network can be implemented in different topologies and include only a part of the standard model levels. To assess the applicability of each network, we estimate the volume of messages between the modules and the frequency of their transmission for the worst case. A special mathematical representation is often used for such estimates - network calculus, which is based on min-plus algebra. 
In network calculus, all network nodes are represented as black boxes with multiple inputs and outputs. Each input is characterized by the so-called arrival function (arrival curve), and each output - departure function (departure curve). Both of these functions characterize the total amount of information received or sent by a particular host. Some of the networks are more amenable to such formalism than others. For example, when using networks with priority of delivery such as CAN, must make extra efforts to analyze traffic due to the fact that the message with higher priority to interrupt the transmission of a message with lower priority [24]. To evaluate the performance of the network as a whole, you must first evaluate the parameters of the arrival curves for each transmitter in each module and submodule. Let us use the Heaviside function (1) to set the step curve (2), which is the arrival curve for the submodule transmitter (PM), where $f_{s m}$, $\mathrm{Hz}$, is the frequency, a $V_{s m}$, bytes, is the payload volume of the transmitted messages.

$$
\begin{aligned}
& h(t)=\left\{\begin{array}{l}
0, x<0 \\
1, x \geq 0
\end{array}\right. \\
& A_{s m}(t)=\sum_{n=0}^{\infty} V_{s m} \cdot h\left(\mathrm{t}-\frac{n}{f_{s m}}\right)
\end{aligned}
$$

Let's evaluate these parameters for various modules and submodules:

1. For transport module (see fig. 2):

1.1. For drive submodules: $f_{s m} \geq 100 \mathrm{~Hz}$, new speed set point $V_{s m}=4$ bytes, readings on the current speed and position of the output shaft $V_{s m}=8$ bytes. There can be several such submodules.

1.2. For collision elimination sensors submodules: $f_{s m} \geq 100 \mathrm{~Hz}$, sensors can be two kinds: (1) bumper with touch sensors $V_{s m}=1$ bytes, (2) non-contact distance sensor $V_{s m}=4$ bytes. There can be several such submodules.

1.3. For the motion control submodule: $f_{s m} \geq 100 \mathrm{~Hz}$, the submodule is given the desired linear and angular velocities $V_{s m}=8$ bytes, the actual position of the module relative to a certain coordinate system $V_{s m}=4 \cdot 6=24$ bytes.

2. For sensor module (see fig. 3):

2.1. For a dynamic search obstacles submodule: $f_{s m} \sim 1 \mathrm{~Hz}$, this submodule can transmit the segmented view of environment in either of $n$ line segments in the plane $V_{s m}=n \cdot 16$ bytes or $n$ line segments in space $V_{s m}=n \cdot 16 \cdot 6=$ $n \cdot 48$ bytes. There can be several such submodules.

2.2. For sensory information processing submodule: $f_{s m} \sim 1 \mathrm{~Hz}$, this sub-module processes signals from various sensors and generates the average estimate of the distance $V_{s m}=8$ bytes.

3. For power module (see fig. 4):

3.1. For the generalized power management submodule: $f_{s m} \sim 1 \mathrm{~Hz}$, this submodule periodically publishes information about the current state of the battery, the maximum load current, etc $V_{s m}=1 . .4 \cdot 1 . .4$ bytes.

In the above data, all frequency parameters $f_{s m}$ depend on the speed of the robot. For other modules, the pattern will be approximately the same. Actuating modules, such as transport module, manipulator module or process module (capture module), have high frequencies of interaction inside the module with small sizes of transmitted messages. Sensor modules or data processor modules, on the other hand, have a much lower frequency of message transmission, which is compensated by the large volume of these messages. A separate case is the power module - on the one hand, this module should not very often publish sensor readings, and on the other hand - should quickly convey a message about the need to start a safe shutdown to all modules. Thus, it is important that messages in the communication network are transmitted with the least overhead (overhead), and that all packets have a roughly equal chance of being transmitted despite their size, i.e. that long packets do not occupy the common bus for too long.

It can also be noted that different topologies may be preferred for different module types. Topology "bus" is preferable to the modules of the actuators, especially if they are the creation of horizontal linkages. The star topology is preferable in the case of sensor modules, as it allows data to be transmitted directly to the supervisor. The definition of requirements for the use of certain topologies for different types of networks will be formulated in further research.

Let's examine as the most popular networks Ethernet in star topology and CAN network in bus topology. In network calculus, it's necessary to consider the worst-case bitrates and framerates to estimate the departure functions of the networks. In the following example both networks are regarded as simple first-in-first-out black boxes with fixed bitrate without any consideration of their special collision rules. This method will help avoid some harder modeling tasks for CAN, as discussed earlier and shown in [24]. CAN bus has typical bitrates of 0.5..1 Mbit/second, Ethernet has bitrate of $10 \mathrm{Mbits} / \mathrm{second}$.

To estimate worst-case scenario, let's consider non-optimal fill of CAN bus: extended frames with 7 bytes payload, 3 of which are protocol overhead, which in turn is 119 bits per frame. Also we need to take into account bit-stuffing andinter-frame pauses in CAN transmission, which further increases the frame size by about 1.5, which is 182 bits per frame. 
This coefficient also takes into account any imperfections of CAN bus hardware and software, interacting with it. The resulting departure curve (2) for CAN with bitrates of 0.5 and $1 \mathrm{Mbit} /$ second is shown on fig. 5a.

As for the Ethernet, packets were considered to transmitted over UDP protocol, used by many existing frameworks, with the same byte structure as CAN packet -5 bytes per header and 7 bytes of payload, 3 of which are protocol overhead. Transmitting such small frames is highly inefficient, so they should be packed together in a collection of around 35 frames, comprising a data frame of 420 bytes (near maximum data frame size on most Ethernet implementations), resulting in 140 bytes of payload per frame. Adding header of 46 bytes and inter-frame silence of 12 bytes further increases the frame size to 478 bytes. As considering worst-case scenario, we need to account for collisions, which leave only around $1 / 3$ of useable bandwidth, which turns to be 784 frames/second on $10 \mathrm{Mbit} / \mathrm{second} \mathrm{link.} \mathrm{The} \mathrm{departure} \mathrm{curve} \mathrm{(2)} \mathrm{for} \mathrm{this} \mathrm{setup}$ is shown on fig. $5 \mathrm{~b}$.

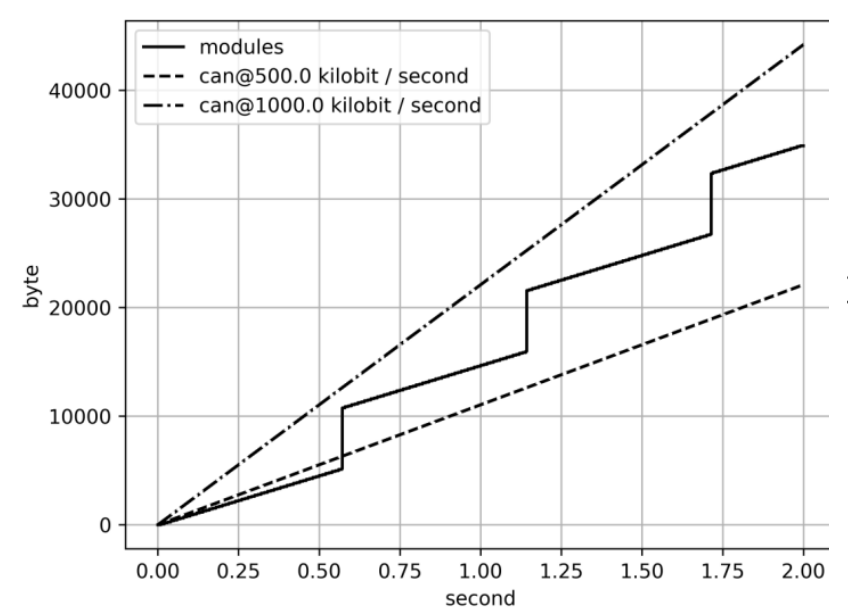

a)

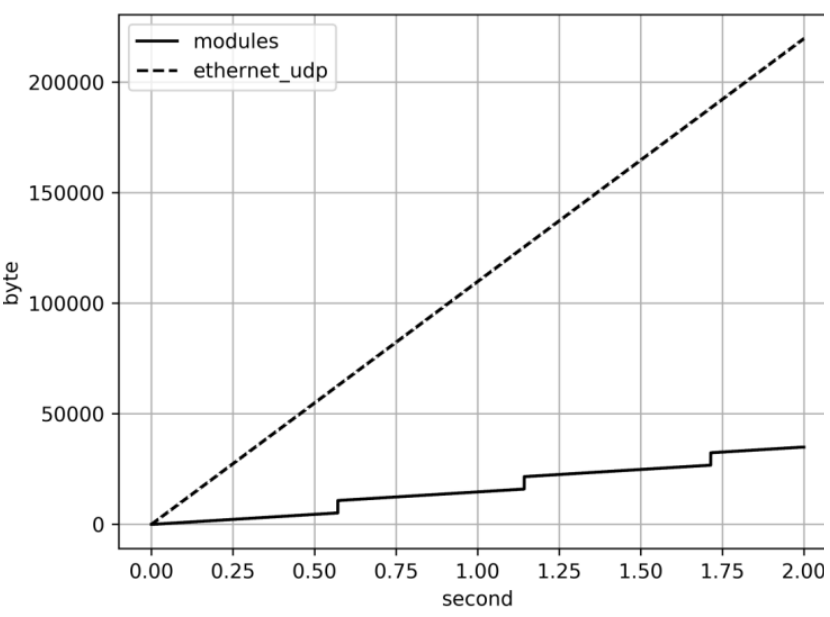

b)

Fig. 5. Departure and arrival curves for CAN with various bitrates (a) and Ethernet (b) networks.

As an example, let's simulate a simple modular robot, on which all networks are united on the same bus. This example MR consists of 4 drive SMs, 4 distance collision elimination SMs, 1 bumper - touch collision elimination SM, 1 motion control module, 2 planar dynamic obstacles search SMs reporting up to 100 line segments, 1 space dynamic obstacles search SM reporting up to 100 line segments and 1 sensory information processing and location module. Each of them forms an arrival curve (2) for respective networks as discussed earlier. For both fig. $5 \mathrm{a}$ and $5 \mathrm{~b}$, we assume that the network is always filled with data to full capacity even in times, when it is not transmitting modules' data. The modules' curve is the sum of all arrival functions (2) of all modules. The main slope is formed by drive modules and submodules. The big steeps are due to dynamic obstacles search SMs high volumes of data transfer, which occur in relatively rare spikes.

In this example, modules utilize almost all bandwidth on higher-speed CAN and a small portion of bandwidth on Ethernet link even in worst-case scenario. But, as discussed earlier, when designing the system, the speed is a major, but not the only criteria, which should be considered. This example also shows the need to split the networks of different modules as this will allow multiplying the bandwidth of any lower-level network by the number of modules, comprising the robot.

\section{Conclusion}

The representation of the robot control system with modular architecture in the form of a multi-level hierarchical (pyramidal) network topology will solve the problem of providing real-time mode, ease the reconfiguration and scaling of the robotic system. The presented studies in the field of intermodule networking have touched only a small part of this problem of such a complex structure.

It is shown that in the first division of CS into functional modules and submodules, it is possible to distinguish at least 3 levels of interaction and subordination, on each of which interaction tasks are defined. In this case, each of the modules and submodules is essentially a specialized edge router that converts network traffic from a higher level to a lower level network traffic. Therefore, in this structure it is possible to use known methods of both software and hardware implementation of routing of information flows as well as define new ways, more suitable for robotic applications.

Since the multilevel topology implies the separation of full-functional modules into simpler submodules, the structure of intermodule information interaction should provide for the transfer of changes in the mass-dimensional parameters of the modules in the case of reconfiguration of the robot.

Most embedded computing devices, which use is a prerequisite in multi-level CS, have a CAN interface. Therefore, special attention was paid to this standard in the above studies. But, as CAN has a rather low bandwidth, therefore a faster Ethernet is also examined. Both standards were compared to the actual needs in terms of informational distribution of various modules. 
In the future, it is necessary to conduct a more detailed analysis of the applicability of different networks, such as I2C, SPI, RS-232, RS-485, CAN, USB, Ethernet, EtherCAT, at different levels of the hierarchy. You must define the requirements for network resources at different levels of the hierarchy and the restrictions on their use. The main goal of further research is to create a new, universal specification for the intermodule interaction method that could work with any coding method and in any communication network.

\section{Acknowledgments}

Research is supported by the Russian Foundation for Basic Research: Grant 19-07-00892a.

\section{References}

[1] Andreev, V. \& Poduraev, Y. (2016). Network-Based Design of Heterogeneous Modular Mobile Robotic Systems, Proceedings of the 27th DAAAM International Symposium, pp.0004-0009, B. Katalinic (Ed.), Published by DAAAM International, ISBN 978-3-902734-08-2, ISSN 1726-9679, Vienna, Austria. DOI: 10.2507/27th.daaam.proceedings.001.

[2] Andreev, V.; Kim, V. \& Pletenev, P. (2017). The Principle of Full Functionality - the Basis for Rapid Reconfiguration in Heterogeneous Modular Mobile Robots, Proceedings of the 28th DAAAM International Symposium, pp.0023-0028, B. Katalinic (Ed.), Published by DAAAM International, ISBN 978-3-902734-11-2, ISSN 1726-9679, Vienna, Austria. DOI: 10.2507/28th.daaam.proceedings.003.

[3] Andreev, V. \& Kim, V. (2016). Control System and Design of the Motion Module of a Heterogeneous Modular Mobile Robot, Proceedings of the 27th DAAAM International Symposium, pp.0586-0594, B. Katalinic (Ed.), Published by DAAAM International, ISBN 978-3-902734-08-2, ISSN 1726-9679, Vienna, Austria. DOI: 10.2507/27th.daaam.proceedings.086.

[4] Andreev V.P., Kim V.L., Pletenev P.F. (2018). Hardware \& Software Solution for Rapid Reconfiguration of Heterogeneous Robots, Mekhatronika, Avtomatizatsiya, Upravlenie, 2018, vol.19, no.6, pp.387-395. DOI: 10.17587/mau.19.387-395.

[5] Andreev, V.P. (2019) The concept of using the theory of multi-agent systems to design control systems for mobile robots with modular architecture. Extreme robotics // Proceedings of the International Scientificand Technological Conference. -Saint-Petersburg, in press

[6] Andreev V.P., Kim V.L. Modular architecture of a mobile robot transport platform for a motion task on a rough terrain. Extreme robotics // Proceedings of the International Scientificand Technological Conference. -SaintPetersburg, in press.

[7] Andreev, V. \& Tarasova, V. (2018). Identification of the Obstacle Shape Using the Ultrasonic Sensors Module of Modular Mobile Robot, Proceedings of the 29th DAAAM International Symposium, pp.1001-1009, B. Katalinic (Ed.), Published by DAAAM International, ISBN 978-3-902734-20-4, ISSN 1726-9679, Vienna, Austria. DOI: 10.2507/29th.daaam.proceedings.143.

[8] Mayoral, V.; Hernandez, A.; Kojcev, R.; Muguruza, I.; et al. (2017). The shift in the robotics paradigm - the Hardware Robot Operating System (H-ROS); an infrastructure to create interoperable robot components, NASA/ESA Conference on Adaptive Hardware and Systems (AHS), Pasadena, CA. - 2017. - pp.229-236.

[9] Herbrechtsmeier, S.; Korthals, T.; Schopping, T. \& Ruckert, U. (2016). AMiRo: a modular \& customizable opensource mini robot platform. 20th International Conference on System Theory, Control and Computing (ICSTCC), Sinaia. - 2016. - pp.687-692.

[10] Bonarini, A.; Matteucci, M.; Migliavacca, M .\& Rizzi, D. (2014). R2P: An open source hardware and software modular approach to robot prototyping. Robotics and Autonomous Systems. - 2014. - No.62. - pp.1073-1084.

[11] Losada, D.P.; Fernández, J.L.; Paz, E. \& Rafael Sanz (2017). Distributed and modular CAN-based architecture for hardware control and sensor data integration. Sensors. - 2017. - No.17. - pp.1013-1030.

[12] Peng, L.; Guan, F.; Perneel, L.; Fayyad-Kazan, H. \& Timmerman M. (2016) EmSBoT: A lightweight modular software framework for networked robotic systems. 2016 3rd International Conference on Advances in Computational Tools for Engineering Applications (ACTEA), Beirut, 2016, pp.216-221. doi: 10.1109/ACTEA.2016.7560142.

[13] Pletenev P. F. (2017) 1/PIMI - Protocol of intermodular interaction Available at: https://asmfreak.github.io/modular_robots_rfc/1/ПMMB/. Accessed: 20.01.2017.

[14] Andreev, V.P. \& Pletenev P.F. (2018) Method of information interaction for distributed control systems of robots with modular architecture. SPIIRAS Proceedings. 2018. № 57 (2). pp.134-160.

[15] GOST R ISO/MEK 7498-1-99. — «Information technology. Open systems interconnection. Basic reference model. Part 1. The basic model». — OKS: 35.100.70. — In use since 01.01.2000. — 62c.

[16] Murata, S.; Yoshida, E.; Kamimura, A.; Kurokawa, H.; Tomita, K. \& Kokaji, S. (2002). M-TRAN: selfreconfigurable modular robotic system, IEEE/ASME Transactions on Mechatronics. - 2002. - No.7(4). - pp. 432-441.

[17] Østergaard, E.H.; Kassow, K.; Beck, R. \& Lund, H.H. (2006). Design of the ATRON lattice-based selfreconfigurable robot, Autonomous Robots. - 2006. - No.21(2). - pp.165-183. 
[18] Guifang Qiao; Guangming Song; Jun Zhang; Hongtao Sun; Weiguo Wang \& Aiguo Song (2012). Design of Transmote: a Modular Self-Reconfigurable Robot with Versatile Transformation Capabilities, Proceedings of the 2012 IEEE International Conference on Robotics and Biomimetics, 2012. - pp.1331-1336.

[19] Toshio Fukuda; Tsuyoshi Ueyama; Yoshio Kawauchi; Fumihito Arai (1987). Concept of cellular robotic system (CEBOT) and basic strategies for its realization, Computers Elect Engng. 1987. vol.18, no.1. - pp.11-39.

[20] Baca, J.; Ferre, M. \& Aracil R. (2012). A heterogeneous modular robotic design for fast response to a diversity of tasks, Robotics and Autonomous Systems. 2012. Vol.60. No.4. - pp.522-531.

[21] Lyder, A.H.; Stoy, K.; Mendoza-Garcia, R.F.; Larsen, J.C. \& Hermansen, P. (2013). On sub-modularization and morphological heterogeneity in modular robotics, Intelligent Autonomous Systems of Advances in Intelligent Systems and Computing. Springer Berlin Heidelberg. 2013. Vol.193. No.12. - pp.649-661.

[22] Hancher, M.D. \& Hornby, G.S. (2006). A modular robotic system with applications to space exploration, 2nd IEEE International Conference on Space Mission Challenges for Information Technology (SMC-IT'06). Pasadena, CA: Publisher «IEEE». 2006. - pp.132-140.

[23] Mendoza-Garcia, R.-F.; Lyder, A.; Christensen, D. J. \& Stoy, K. (2009) Reusable Electronics and Adaptable Communication as Implemented in the Odin Modular Robot. 2009 IEEE International Conference on Robotics and Automation - P. 1152-1158.

[24] Klehmet, U.; Herpel, T.; Hielscher, K. \& German, R. (2008) Delay Bounds for CAN Communication in Automotive Applications, 14th GI/ITG Conference - Measurement, Modelling and Evalutation of Computer and Communication Systems, Dortmund, Germany, 2008, pp. 1-15. 